\title{
A Taxonomy of WiFi Sensing: CSI vs Passive WiFi Radar
}

\author{
W. Li*, M. J. Bocus ${ }^{\dagger}$, C. Tang*, S. Vishwakarma*, R. J. Piechocki ${ }^{\dagger}$, K. Woodbridge ${ }^{\ddagger}$, K. Chetty* \\ *Department of Security and Crime Science, University College London, UK \\ ${ }^{\dagger}$ Department of Electrical and Electronic Engineering, University of Bristol, UK \\ ${ }^{\ddagger}$ Department of Electronic and Electrical Engineering, University College London, UK \\ \{wenda.li, k.woodbridge, k.chetty, chong.tang.18, s.vishwakarma\}@ucl.ac.uk, \\ \{junaid.bocus,r.j.piechocki\}@bristol.ac.uk
}

\begin{abstract}
WiFi sensing has shown promising potentials in a number of applications such as healthcare, smart transportation and home automation. Human activity recognition without the use of any cooperative device such as phones or wearable technologies can be achieved by two WiFi based approaches: Channel State Information (CSI) and Passive WiFi Radar (PWR). CSI systems rely directly on WiFi as a communications system, whilst PWR treats the WiFi signal as an illuminator for use in a radar signal processing. However, there has not been a comprehensive comparative study on the similarities and differences between the two systems. To examine the performance of both systems we implement two hardware platforms for CSI and PWR, and use them concurrently to capture the human movements. In this paper, we present Doppler measurements from the two systems and compare their performance using a dataset obtained from five subjects undergoing six activity classes. It is observed that both systems have very different Doppler signatures, and are sensitive to the transmitter-target-receiver geometries. CSI has a better performance in Line-of-Sight $(\mathrm{LoS})$ configurations, whereas PWR has better performance in bistatic configurations where the WiFi access point and radar receiver are spatially separated. It is envisioned that a more robust system should leverage strengths of both the CSI and PWR systems jointly to maximize their benefits in wireless sensing.
\end{abstract}

Index Terms-Activity Recognition, CSI, Passive WiFi Radar, Wireless Sensing

\section{INTRODUCTION}

The increasing popularity of wireless devices has resulted in WiFi experiencing a global and rapid growth. Recent research has shown that wireless signals can also be used for shortrange detection [1]. The main idea behind WiFi sensing is that a moving person will affect the communication channel of a WiFi signal in terms of frequency shift, propagation paths and signal attenuation [2]. As a result, the communication channel becomes time-varying with the human activities and hence we can exploit this fact for monitoring purposes. Compared to other technologies used in activity recognition such as camera systems and wearable sensors, WiFi-based sensing technology does not raise privacy concerns, operates in all weather and light conditions in contactless manner, and is widely available in almost all indoor environments. Factors such as these make the technology a highly attractive solution for real-world usecases.

In the field of activity recognition using wireless sensing, there are two major approaches, namely, the Channel State
Information (CSI) system [1] and Passive WiFi Radar (PWR) [3]. Both CSI and PWR use the same signal source and have the same function but they have different working mechanisms: CSI system are an extension of WiFi communications, whereas the PWR system is based on the principles of radar. CSI is used to estimate the communication channel between the transmitter and receiver, and provides both amplitude and phase information. With appropriate radio hardware, the phase information can be used to extract the Angle of Arrival (AoA), Time of Flight (ToF) and Time Difference of Arrival (TDoA). Previous work has demonstrated the feasibility of CSI in activity recognition [4], finger gesture recognition [5], crowd counting [6] and fall detection [7]. In comparison, a PWR system correlates the transmitted signal from a WiFi Access Point (AP) and reflected signal from the surveillance area. It calculates the relative distance and velocity between the object and antenna. However, due to the relatively low bandwidths of existing WiFi protocols, the range resolution for a PWR system is rather coarse and as a result most applications make use of the Doppler output, which is of a very high resolution owing to the long integration times possible with receiveronly systems. Moreover, as future WiFi standards evolve to incorporate higher bandwidths e.g. 802.11ac and 802.11ad the range resolutions of these systems will move towards sub-meter precision opening-up new possibilities for indoor sensing. PWR systems have already been used in several sensing applications ranging from through-wall [3] and human presence detection [8], to detecting signs-of-life [9].

In this paper, we carry out a comparative study of CSI and PWR systems. We first outline the key operational concepts of the two systems when processing WiFi signals. To verify the concepts between the two systems, we have implemented two hardware platforms to demonstrate and compare the performance of each system for activity recognition. The CSI system is implemented using the popular Intel 5300 Network Interface Card(NIC) [10], and the PWR system is built using a SoftwareDefined-Radio (SDR) [11] platform. We collect the Doppler measurements from both systems for six different activities classes across five human subject. Compared to previous work on bot CSI [4]-[7] and PWR [3], [9], [12], this work makes the following contributions: 
- To the best of the authors' knowledge, this is the first work that compares the performance and outlines the difference between the CSI and PWR systems for activity recognition.

- The data sets generated in this work were collected by a two separate CSI and PWR systems but were operated simultaneously with synchronized measurements.

- Experimental results have shown that the two systems have different performance in terms of Doppler spectrograms, system geometry and coverage area.

This paper is organized as follow: An overview of the CSI and PWR systems is given in Section II; The signal processing is described in Section III; Experimental results from a real environment are presented in Section IV and conclusions are drawn in Section V.

\section{System OVERVIEW}

\section{A. WiFi Signal}

OFDM is widely used in many WiFi standards such as IEEE $802.11 \mathrm{a} / \mathrm{g} / \mathrm{n} / \mathrm{ac}$. The bandwidth in an OFDM system is shared among multiple overlapping but orthogonal subcarriers. The transmitted OFDM signal is defined as:

$$
x(t)=\frac{1}{\sqrt{N}} \sum_{n=1}^{N} a_{n} e^{j \frac{2 \pi}{T_{s}} n t}
$$

where $N$ is the number of subcarriers, $a_{n}$ is the $n$th symbol in the constellation symbol sequence such as QPSK or QAM, and $T_{s}$ is the OFDM symbol period. The received signal $y(t)$ includes both the multipath reflections and the direct signal. These reflections from a moving person and surrounding stationary objects can be represented as a summation of the delayed and phase shifted transmitted signal as follows:

$$
y(t)=\sum_{p} A_{p} e^{j 2 \pi f_{d} t} x(t-\tau)+n(t),
$$

where $A_{p}, \tau, f_{d}$ are the attenuation factor, delay and Doppler shift for the $p$-th path, respectively, and $n(t)$ is Additive White Gaussian Noise (AWGN). The CSI signal, $H\left(f_{c}, t\right)$, at carrier frequency $f_{c}$ can then be calculated as $H\left(f_{c}, t\right)=$ $Y\left(f_{c}, t\right) / X\left(f_{c}, t\right)$. Although the mechanisms of CSI and PWR are different, however, the key idea of the two systems is to capture the changes in the wireless signal caused by the moving personnel, and convert these into Doppler spectrograms.

\section{B. Overview of CSI and PWR System Model}

In this work, we have implemented two separate systems. The overall system models for the CSI and PWR systems are shown in Fig 1. The CSI system uses an Intel 5300 NIC [10] to calculate the raw CSI signal based on a pre-defined preamble sequence in each WiFi packet. The PWR system uses the USRP-2921 [11] to acquire the raw wireless signal and has no knowledge about the transmitted WiFi signal or router. The PWR system treats the WiFi signal as a third-party signal.

There are many different types of signal processing for the CSI system depending on the intended application [5]-[7]. Since we focus on activity recognition in this work, we decide to use the Doppler spectrogram as the measurement, as it can present meaningful information about the activity and it is also insensitive to static objects in the background. The signal processing for the CSI system consists of three parts. In the first step, we adopt the Discrete Wavelet Transform (DWT) technique to filter out in-band noise and preserve the high frequency components. The next step is to reduce the size of the massive CSI data using the Principal Component Analysis (PCA), since the raw CSI data is logged for 30 subcarriers and 3 receive antennas at a packet rate of $1 \mathrm{kHz}$. The final step is to convert the PCA values into Doppler spectrograms using the Short-Time Fourier Transform (STFT).

The signal processing for the PWR system also includes three parts. Firstly, a Cross Ambiguity Function (CAF) is used to generate a range-Doppler surface based on the transmitted and reflected signals. Secondly, a CLEAN algorithm [3] has been used to suppress the direct signal from transmitter which is the major interference for the PWR system. Lastly, a Constant False Alarm Rate (CFAR) is used to detect the noise that is generated during the CAF process to further improve the spectrogram quality.

In the CSI system, we collect the raw CSI data and store it for offline processing. In the PWR system, we use a lowcomplexity design [13] which can process the raw WiFi signal and output the Doppler spectrogram in real-time. Details about each signal processing block are given in Section III.

\section{Understanding the Mechanisms}

The mechanisms in time and frequency domains for the CSI and PWR systems are presented in Fig 2. A complete WiFi packet includes a preamble which is used to estimate the channel and the data signal which carries the information for communication. There is also a time gap (of varying length) between two adjacent WiFi packets. As it can be seen in Fig 2 , in the time domain, the CSI system does not take full advantage of a WiFi packet, since it only uses the preamble signal and ignores the data signal. In comparison, the PWR system has no knowledge about the WiFi packet regarding the preamble or data signal. The PWR system usually captures a signal of much longer duration than the CSI system to ensure sufficient amount of WiFi signal can be captured. As a result, the PWR system uses the entire WiFi signal, however, it also captures the time gap which could be a potential noise source.

In the frequency domain, the CSI system calculates the channel information from each subcarrier. These measurements can provide fine-grained features but depending on the number of transmit and receive antennas as well as the packet rate, they normally result in a considerable amount of data. On the other hand, the PWR system does not process the signal for each subcarrier but treats the entire OFDM symbol as one signal. For this reason, the PWR system cannot access the information within each individual subcarrier. The bandwidth for the PWR system is adjustable depending on the requirements and is limited by the computational power. A $20 \mathrm{MHz}$ bandwidth implies that 20 million data points need to be processed every second which is beyond the processing capability of a laptop. In this work, the PWR system uses only a portion of the WiFi spectrum. 


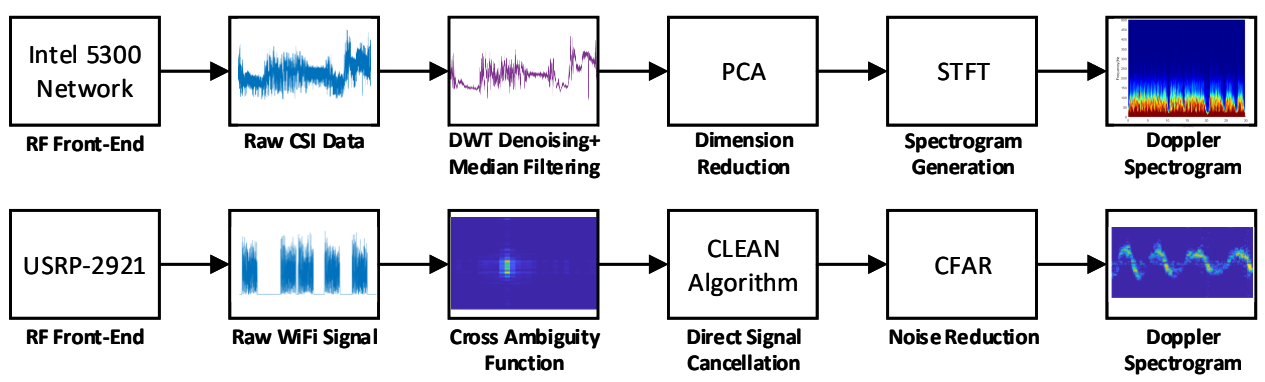

Fig. 1: Block diagram overview of CSI (top) and PWR (bottom) systems

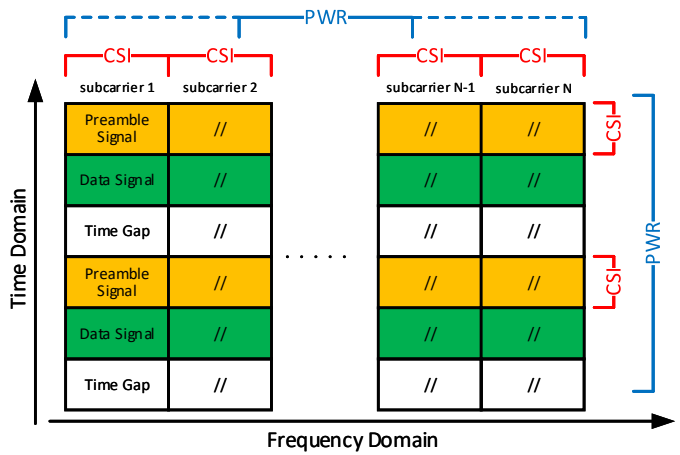

Fig. 2: Block diagram overview of CSI (top) and PWR (bottom) systems

\section{Signal Processing}

This section presents the signal processing techniques used in this work, including noise reduction, dimension reduction, spectrogram generation in the CSI system and cross ambiguity function, CLEAN algorithm and CAR in the PWR system.

\section{A. CSI System}

1) Denosing and Smoothing: The raw CSI data is noisy in nature due to the power and data rate changes in the WiFi AP. Therefore, the DWT technique has been used to filter out high frequency components and burst noises, while avoiding significant distortion to the signal. The raw CSI signal is passed through a set of high pass and low pass filters at each level. The output from the high pass and low pass filters provides the detailed and approximation coefficients, respectively. These detailed coefficients in the first level contains information about the noise and the abrupt changes caused by human activity. Therefore, the detailed coefficients in the first level are used to compute a threshold. The latter is adapted for lower wavelets and the noise is removed in all levels without introducing significant distortion to the signal. Afterwards, a 1-D median filter has also been applied to the signal to remove any undesired transients in the CSI measurement which are not caused by human motion.

2) Dimension Reduction in CSI measurement: The raw CSI measurement collected from the Intel 5300 NIC results in a significant amount of data. In this work, the CSI system consists of one transmitting and three receiving antennas with a packet rate of $1 \mathrm{kHz}$ and this results in $1 \times 3 \times 30 \times 1 k=90 k$ complex CSI values per second. The size of this data is too large to be directly used as input to a machine learning algorithm for classification. Therefore, dimension reduction is required in the CSI system to reduce the overall computational complexity. We adopt the PCA technique to identify the timevaring correlations between the CSI streams which are then combined to extract components that represent the variation in the CSI measurements. The number of principal components is selected to acquire a trade-off between classification performance and computational complexity [14]. Usually, the first two or three principal components capture $70 \sim 80 \%$ of the signal variance. Similar to [14], we extract the first six principal components for the CSI system. However, we discard the first one since it contains noise due to the reflection from the stationary objects in the background. Therefore, only the next five principal components are used for further processing.

3) Spectrogram Generation: CSI measurements are highly sensitive to the surrounding environment and radio-frequency reflections from the human body exhibit different frequencies when performing different activities. Previous work [4] requires a calibration process to scan the background. To avoid this, we convert the PCA signal into spectrograms using the STFT. STFT applies a sliding window to obtain equally-sized segments of the signal and then performs FFT on the samples in each segment. The STFT of a time-domain input signal $x[n]$ can be represented as [15]:

$$
X(t, k)=\sum_{n=-\infty}^{\infty} x[n] w[n-t] e^{-j k n}
$$

where $k$ denotes the frequency index, and $w[n]$ represents a window function (e.g., Hamming window). The output spectrogram contains three dimensions, namely, time, frequency, and amplitude of FFT. The CSI spectrogram is generated from the five principal components which are then averaged to obtain the final spectrogram.

\section{B. PWR System}

1) Cross Ambiguity Function: The CAF is an effective tool used in passive radar field to extract target range $\tau$ and Doppler $f_{d}$ information. It requires two channels, a surveillance channel $S_{\text {sur }}(t)$ collects the signal from the surveillance area, while a reference channel $S_{r e f}(t)$ measures the signal 
directly from the transmitter. Due to the limited bandwidth $B$ of a WiFi signal, the range resolution $\Delta \tau=C / 2 B$ is not sufficient for indoor applications (between 7.5 to 1.875 meters). Doppler resolution is defined by the integration of time $T_{i}$ as $\Delta f_{d}=1 / T_{i}$. This allows the Doppler resolution to be adjusted for detecting human activities. In this work, we use a low-complexity version of CAF [13] which divides a long sequence into several short batches so that the processing can be faster. The low-complexity CAF equation can be written as:

$$
C A F\left(\tau, f_{d}\right)=\int_{0}^{T_{i}} x(t) y *(t-\tau) e^{j 2 \pi f_{d} t} d t
$$

where $*$ denotes a complex conjugate operation, $N_{b}$ is the number of batches, $T_{b}$ is the batch length and $n$ is the index of the beacon.

2) Direct Signal Interference Cancellation: The main interference source for a PWR system is the direct signal from the WiFi AP that goes directly to the surveillance channel. This unwanted direct signal contains a higher energy than the signal reflected from a moving target, and can mask the Doppler pulse in the CAF surface. We employ a modified-CLEAN algorithm [3] to remove the direct signal interference from the CAF surface as a means of improving the target signalto-interference ratio. This CLEAN algorithm shares a similar structure to the CAF process but generates a self-ambiguity surface only from the reference channel. It's operation is described below:

$C A F^{k}\left(\hat{\tau}, \hat{f}_{d}\right)=C A F^{k}\left(\tau, f_{d}\right)-\alpha^{k} C A F_{\text {self }}\left(\tau-T_{k}, f_{d}\right)$ where $C A F^{k}\left(\hat{\tau}, \hat{f}_{d}\right)$ is the cleaned surface at the $k$ th iteration, $C A F_{\text {self }}$ is the self ambiguity surface, $\alpha^{k}$ and $T_{k}$ are the amplitude and phase shift of the maximum peak in the $k$ th CAF surface, respectively.

3) Noise Reduction: The last step is to further reduce the noise on the CAF surface. After the CLEAN algorithm, residual noise exists which is attributed to the correlation over the time gap, as shown in Fig 2. Additionally, the CAF surface may be incorrectly processed due to the unstable reference channel that recreates an incomplete transmitted signal. A simple solution is to use CFAR to estimate the background noise distributions and apply to the CAF surface as:

$$
\Lambda=\frac{1}{N_{\tau} \cdot N_{f_{d}}} \sum_{i=1}^{R_{\tau}} \sum_{j=1}^{R_{f_{d}}} C A F\left(\tau_{i}, f_{d_{j}}\right)
$$

where $i$ and $j$ are the indices for range and Doppler bin, respectively, $N_{\tau}$ and $N_{f_{d}}$ are the training length in range and Doppler bin, respectively, and $\Lambda$ is the threshold for CAF surface. The strong pulse above the threshold is representative of human activity, otherwise it is inferred that no motion has occurred.

PWR's Doppler spectrogram is generated by selecting the maximum Doppler pulse from each Doppler bin within a CAF surface, and combined with a number of measurements.

\section{REsults}

In this section, the activity recognition performance is presented. A simple 2D Convolutional Neural Network (CNN)

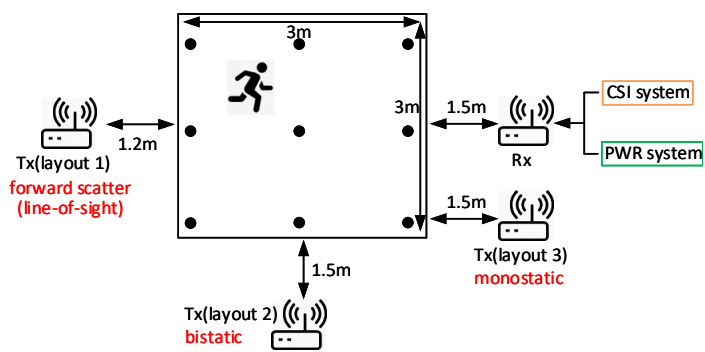

Fig. 3: Experiment layout

has been used as the classifier including one convolutional layer, one max-pooling layer and two fully connected layers. Since the spectrogram sizes from the two systems are different, the parameters used in the two CNNs are also different.

\section{A. Experimental Layout \& Dataset}

To assess and compare the CSI and PWR systems, we carried out several simultaneous measurements to capture the human motions. The experiment layout is shown in Fig 3 with a monitoring area of $3 \mathrm{~m} \times 3 \mathrm{~m}$, with computers and furniture in the surroundings. The receive antenna location was fixed and the transmitter position moved between three different locations. Both systems were operated in the 2.4 $\mathrm{GHz}$ band but were kept on different channels to avoid interference since the CSI system was performing a two-way communication (pinging at $1 \mathrm{kHz}$ ) whereas the PWR system was only receiving. Layout 1 refers to forward scatter (lineof-sight) whereby the transmitter-object-receiver's alignment is around 180 degrees. Layout 2 is when the transmitterobject-receiver is around 90 degrees and this forms a bistatic geometry. Layout 3 is when the transmitter-object-receiver is less than 45 degrees and this is known as the monostatic geometry. Nine positions were tested during the experiment inside the monitoring area, with $1.5 \mathrm{~m}$ separation between the positions.

Subjects were instructed to undertake six different classes activity, namely, walking, sitting, standing from chair, laying down, standing from the floor and picking up small items from the floor. A sliding window has been applied to the Doppler spectrogram with a length of 4 seconds. 5 volunteers of different age groups were involved in this experiment. Each activity was performed in a random fashion with no specific orientation with respect to the receiver antenna. We have collected a total of 1,122 data samples.

\section{B. Comparison of Spectrograms}

The system layout has a significant impact on the WiFi sensing performance. Subjects were asked to walk periodically forward and backward in one direction. Doppler spectrograms from all three different layouts were then generated as illustrated in Fig 4. Spectrograms from the CSI system have similar Doppler signatures with strong pulse across the entire frequency. The high frequency represents the fast torso movements and the low frequency relates to the limb movements. In 

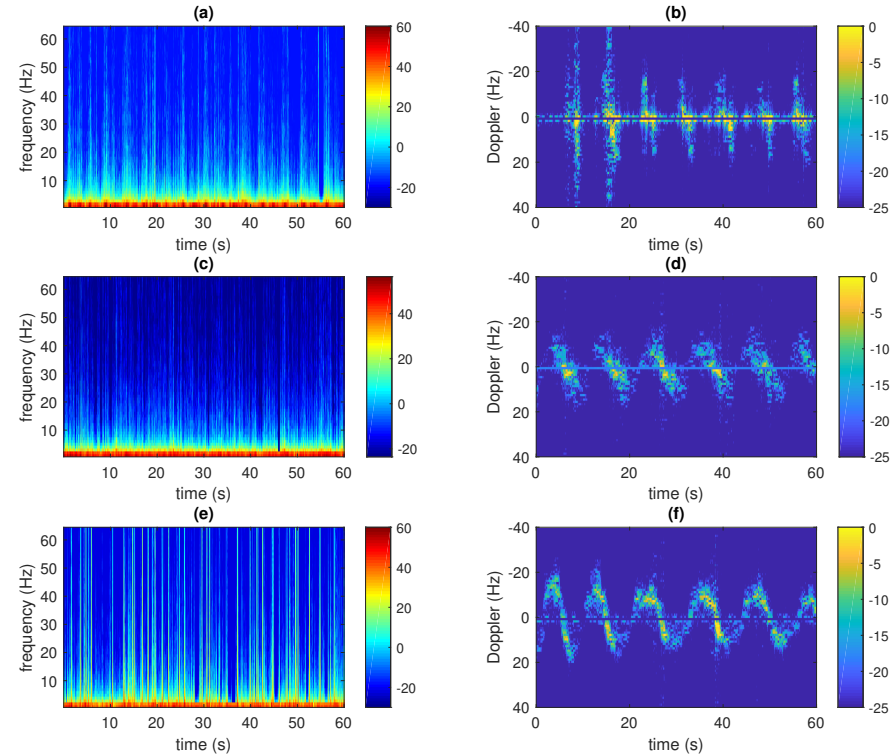

Fig. 4: Walking spectrogram from CSI in (a) layout 1, (b) layout 2, (c) layout 3 and from PWR in (d) layout 1, (e) layout 2, (c) layout 3

comparison, spectrograms from the PWR system have a more varied Doppler profiles which resemble a sinusoidal-wave. The PWR spectrogram in layout 1 (Fig 4(b)) shows low Doppler shift due to the relative velocity between the transmitterobject and object-receiver is almost zero on the line-of-sight. The PWR spectrogram in layout 3 (Fig 4(f)) has the best Doppler signature with the highest Doppler shit and clear shape. Therefore, it is expected there will be some difference in recognition accuracy for the different system layouts. In addition, CSI spectrogram does not contain information about the walking direction, while PWR's spectrogram can show the velocity and direction. This is because CSI measures the signal over a short period of time, considering the duration of a preamble signal, and this is not long enough to calculate the direction. In comparison, the PWR system uses an integration time of 1 second, which is sufficient to observe the direction of the object.

Fig 5 presents the Doppler spectrograms for the other five activities. As it can be seen, CSI's spectrograms are with lower Doppler frequency compared to that in Fig 4, as the result of relatively slower body motion. For example, the Doppler frequency when standing from chair (Fig 5(c)) has a similar shape to standing from the floor (Fig 5(g)). More patterns can be observed in PWR's spectrograms. For example, standing from chair (Fig 5(d)) and standing from floor (Fig 5(h)) both have a positive Doppler shape due to the upward body movement. Similarly, sitting (Fig 5(b)) and laying down on the floor (Fig 5(f)) both contain a negative Doppler shape due to the downward body movement. Moreover, the picking activity (Fig 5(j)) contains both downward and upward body movements. Therefore, as expected, we can see a negative Doppler shape followed by a positive Doppler shape.
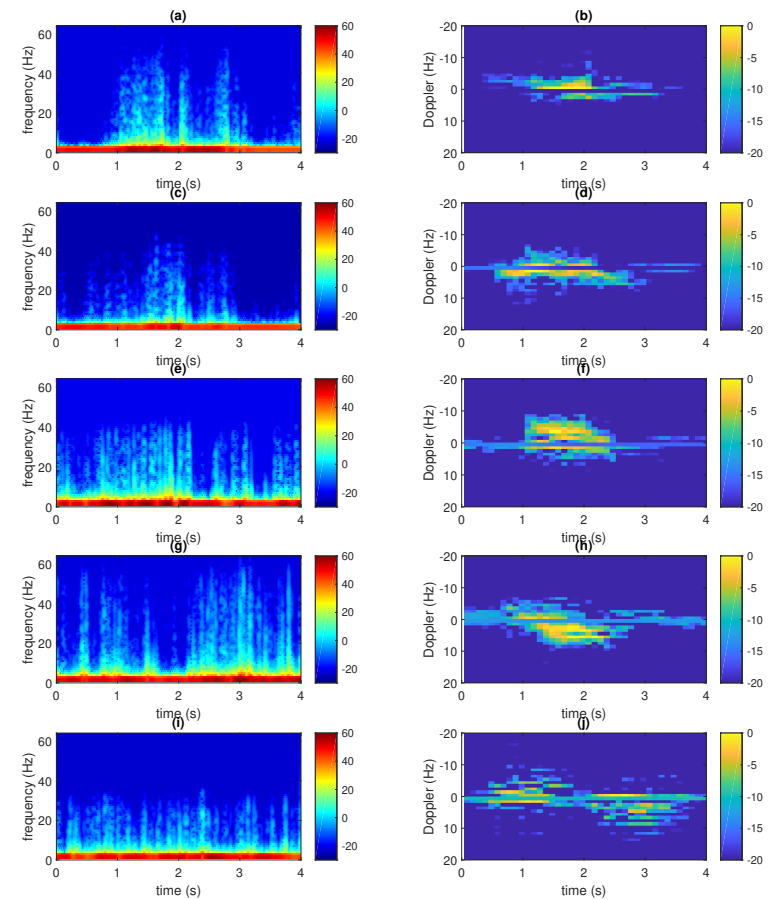

times $_{(\mathrm{h})}^{(\mathrm{s})}$
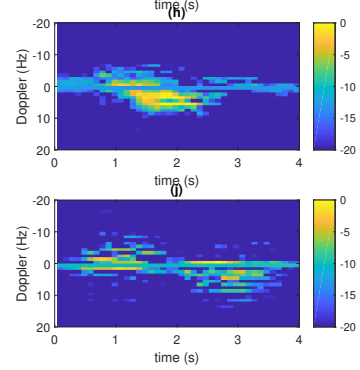

Fig. 5: Spectrograms obtained from layout 2 by CSI system: (a) sitting, (c) standing from chair, (e) laying, (g) standing from floor, (i) picking, and from PWR system: (b) sitting, (d) standing from chair, (f) laying, (h) standing from floor, (j) picking

\section{Recognition Accuracy}

Here we present the activity recognition results from both systems. $80 \%$ of the dataset from all three layouts was randomly chosen and used for training, while the remaining $20 \%$ was used for testing. The confusion matrices are shown in Fig 6. The numbers 1 to 6 in the $\mathrm{x}$ and $\mathrm{y}$ axes refer to the activities walking, sitting, standing from chair, laying, standing from floor and picking up, respectively. The overall accuracy for the CSI system is $67.3 \%$ and the PWR system has a similar accuracy at $66.7 \%$. This accuracy is lower compared to similar previous work such as [4], [13], [14] where an accuracy higher than $85 \%$ has been obtained. However, these studies have only considered a single optimum layout, whereas we have considered three different layouts consisting of a mixture of forward scatter (LoS), bistatic and monostatic layouts (NLoS). Moreover, the change of measurement position results in variations in reflection power and Doppler signature at the receiver side. Both systems have the best recognition result in activity 1 (walking) where the accuracy is more than $90 \%$. This is because the walking activity has higher Doppler shifts/frequencies than other activities for any direction or layout. The second best result is observed for activity 6 (picking up) which is more than $70 \%$, while the other four activities have relatively low accuracy. The CSI system has the worst performance for activity 3 (standing) and activity 5 (standing from floor), whereas the PWR system has the 

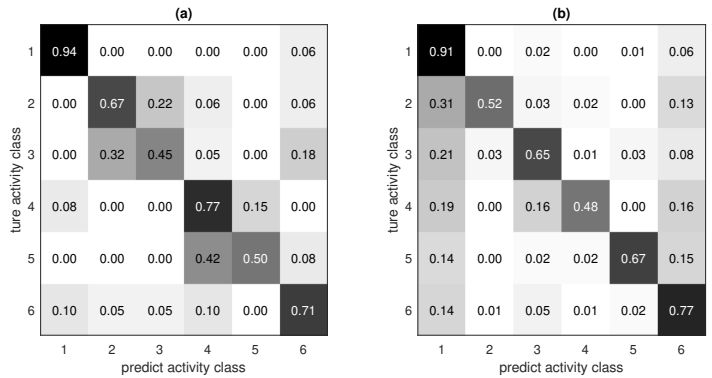

Fig. 6: Confusion matrix on combined result on layout $1,2,3$ from (a) CSI system and (b) PWR system

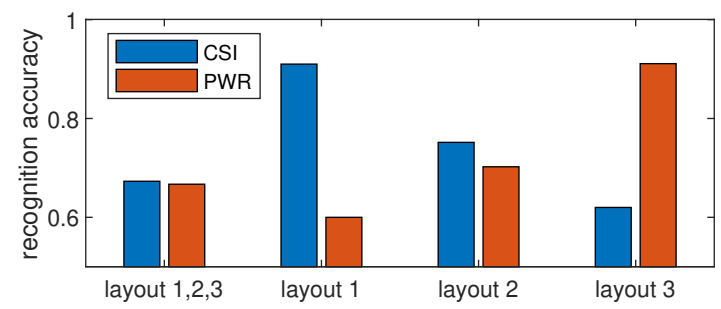

Fig. 7: Recognition accuracy from each layout

worst performance for activity 2 (sitting) and activity 4 (laying down). In addition, most wrong predictions in the CSI system happen between activity 2 (sitting) and activity 3 (standing from chair), and activity 4 (laying) and activity 5 (standing from floor).

To further evaluate the performance in different layouts, we train and test the dataset within each layout. The recognition accuracy is shown in Fig 7. As expected, the CSI system has the best performance in layout 1 with an accuracy as high as $90 \%$ and the worst performance in layout 3 with an accuracy of only $62 \%$. In comparison, the PWR system has the best performance in layout 3 with an accuracy of $91.3 \%$ and worst accuracy in layout 1 at only $60 \%$. Both systems have almost similar accuracy in layout 2 at around $70 \%$ which is still better than the combined accuracy. As mentioned before, the CSI system works better in a forward scatter (LoS) layout, while the PWR system has better performance in a monostatic layout (NLoS). These results demonstrate the coverage of the two systems. The geometry problem represents a challenge for WiFi sensing as in the real world, the distance/angle between target and antenna is not always constant. A multiple channel system with spatially distributed receiver nodes could be a potential solution to address these geometrical limitations. Fusing both types of system would permit data collection from different aspect angles and leverage the advantages that both CSI and PWR systems have to offer to achieve optimal sensing performance.

\section{CONCLUSIONS}

In this paper, we have presented a pilot study on the comparison between two popular types of systems used in WiFi sensing. We have demonstrated how the working mechanism, signal processing and system layouts can all affect the detection performance of the CSI and PWR systems. Those systems have been implemented using different hardware platforms but they were deployed to simultaneously capture the Doppler spectrograms as a result of human motion, which were then used to verify the concepts. Experimental results have shown that the two systems have varied performance in terms of the coverage and layout, where CSI and PWR achieve the best performance in the forward scatter $(\mathrm{LoS})$ and monostatic (NLoS) layouts, respectively.

Future works include the development of a multiple channel system to address the challenge of system geometry. Also, a system which can combine the benefits of the CSI and PWR systems could be a potential solution in WiFi sensing.

\section{ACKNOWLEDGMENTS}

This work was funded under the OPERA Project, the UK Engineering and Physical Sciences Research Council (EPSRC), Grant EP/R018677/1.

\section{REFERENCES}

[1] Y. Ma, G. Zhou, and S. Wang, "Wifi sensing with channel state information: A survey," ACM Computing Surveys (CSUR), vol. 52, no. 3, p. 46, 2019.

[2] R. v. Nee and R. Prasad, OFDM for wireless multimedia communications. Artech House, Inc., 2000.

[3] K. Chetty, G. E. Smith, and K. Woodbridge, "Through-the-wall sensing of personnel using passive bistatic wifi radar at standoff distances," IEEE Transactions on Geoscience and Remote Sensing, vol. 50, no. 4, pp. 1218-1226, 2011.

[4] W. Wang, A. X. Liu, M. Shahzad, K. Ling, and S. Lu, "Understanding and modeling of wifi signal based human activity recognition," in Proceedings of the 21st annual international conference on mobile computing and networking. ACM, 2015, pp. 65-76.

[5] S. Tan and J. Yang, "Wifinger: leveraging commodity wifi for finegrained finger gesture recognition," in Proceedings of the 17th ACM international symposium on mobile ad hoc networking and computing. ACM, 2016, pp. 201-210.

[6] W. Xi, J. Zhao, X.-Y. Li, K. Zhao, S. Tang, X. Liu, and Z. Jiang, "Electronic frog eye: Counting crowd using wifi," in IEEE INFOCOM 2014-IEEE Conference on Computer Communications. IEEE, 2014, pp. 361-369.

[7] H. Wang, D. Zhang, Y. Wang, J. Ma, Y. Wang, and S. Li, "Rt-fall: A real-time and contactless fall detection system with commodity wifi devices," IEEE Transactions on Mobile Computing, vol. 16, no. 2, pp. 511-526, 2016.

[8] W. Li, B. Tan, and R. J. Piechocki, "Wifi-based passive sensing system for human presence and activity event classification," IET Wireless Sensor Systems, vol. 8, no. 6, pp. 276-283, 2018.

[9] — , "Non-contact breathing detection using passive radar," in 2016 IEEE International Conference on Communications (ICC). IEEE, 2016, pp. $1-6$.

[10] "Linux 802.11n CSI tool," https://dhalperi.github.io/linux-80211ncsitool/, (Accessed on 02/11/2020).

[11] $\mathrm{Ni}$ usrp $2920 . \quad$ [Online]. Available: http://sine.ni.com/nips/cds/view/p/lang/en/nid/212995

[12] F. Colone, P. Falcone, C. Bongioanni, and P. Lombardo, "Wifi-based passive bistatic radar: Data processing schemes and experimental results," IEEE Transactions on Aerospace and Electronic Systems, vol. 48, no. 2, pp. 1061-1079, 2012.

[13] W. Li, B. Tan, and R. Piechocki, "Passive radar for opportunistic monitoring in e-health applications," IEEE journal of translational engineering in health and medicine, vol. 6, pp. 1-10, 2018.

[14] W. Wang, A. X. Liu, and M. Shahzad, "Gait recognition using wifi signals," in Proceedings of the 2016 ACM International Joint Conference on Pervasive and Ubiquitous Computing, 2016, pp. 363-373.

[15] K. Qian, C. Wu, Z. Zhou, Y. Zheng, Z. Yang, and Y. Liu, "Inferring motion direction using commodity wi-fi for interactive exergames," in Proceedings of the 2017 CHI Conference on Human Factors in Computing Systems, 2017, pp. 1961-1972. 